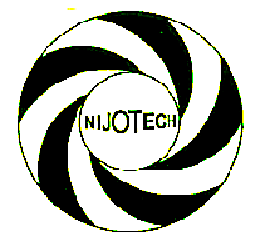

Nigerian Journal of Technology (NIJOTECH)

Vol. 33. No. 1. January 2014 , pp. 33 - 42

Copyright(C) Faculty of Engineering,

University of Nigeria, Nsukka, ISSN: 111 15-8443

www.nijotech.com

http://dx.doi.org/10.4314/nit.v33i1.5

\title{
DEVELOPMENT AND COMPARISON OF DIFFERENT INTENSITY DURATION FREQUENCY MODELS FOR CALABAR, NIGERIA
}

\author{
N. M. Ogarekpe* \\ Department of Civil Engineering, Cross River University of TeChnology, Calabar, NiGERIA.
}

Abstract

E-mail address: nkpaogarekpe@yahoo.com

\begin{abstract}
This paper is aimed at developing and comparing different intensity duration frequency models. Twenty three years peak rainstorm intensity data with their corresponding durations was collected from the Nigerian Meteorological Agency, Calabar, Nigeria. Microsoft Excel software was used to develop exponential, logarithmic and power intensity-duration-frequency models for return period (T) of between 2 years and 100 years using rainfall intensity data for durations of 2, 5, 10, 15, 30, 60, 120, 240 and 320 minutes. A comparison between the predicted results by the three models revealed that the logarithmic model yielded the closest intensity values to the measured intensities. The exponential, logarithmic and power models gave $R$ square values of between 0.022 and $0.858(0.479 \pm 0.214), 0.007$ and $0.848(0.558 \pm 0.197)$ and0.004 and $0.865(0.618 \pm 0.212)$ respectively. This research reveals that the logarithmic model yields comparatively moderate intensity-duration-frequency models that would ensure adequate sizing and forecast of relevant hydraulic structures in the study area.
\end{abstract}

Keywords: Rainfall intensity, rainfall duration, intensity - duration - frequency models, IDF

\section{Introduction}

Rainfall records are required for planning and development of water resources projects [1]. Rainfall intensity values find useful engineering application as input data in many hydrologic design methods like the estimation of design discharge for flood control structures as well as in erosion control studies where they serve as important parameters in the measurement of erosivity index. The need to have reliable estimates of these values for localities have become more urgent now than ever before because of the recent devastation caused by flood in different parts of the world in addition to the challenges currently being posed by uncertainties occasioned by climate change phenomenon. The applicability of rainfall intensity cannot be overemphasized. In the past, researches have been conducted on the effect of rainfall intensity and energy on gully development in northeastern Enugu State [2]

The Intensity - Duration - Frequency (IDF) relationship is a mathematical relationship between the rainfall intensity, the duration and the return period. The rainfall Intensity Duration Frequency (IDF) relationship is one of the most commonly used tools for the design of hydraulic and water resources engineering control structures. The establishment of such relationship was done as early as 1932 [3]. The rainfall intensity-duration-frequency (IDF) relationship is commonly required for planning and designing of various water resource projects [4]. This relationship may be determined through statistical analysis of samples of records at meteorological stations. Okonkwo and Mbajiorgu [5] developed IDF curves for south eastern Nigeria using two methods, graphical and statistical and the results were compared. IDF data obtained from the graphical and statistical methods were very close at lower return periods between of 2 to 10 years, but differ for higher return periods of 50 to 100 years, although the differences were not significant at 5\% level [5]. Quantification of rainfall is generally done using isopluvial maps and intensity-duration-frequency (IDF) curves [6].

The IDF formulas are the empirical equations representing a relationship among maximum rainfall intensity (as dependant variable) and other parameters of interest such as rainfall duration and frequency (as independent variables). There are several commonly used functions found in the literature of hydrology 
applications [6]. The IDF relation is mathematically as follows:

$i=f(T, d)$

Where $\mathrm{I}$ is the intensity ( $\mathrm{mm} /$ minute), T, the return period(years) and $\mathrm{d}$, the duration (minutes)

The IDF relationship exist in several forms however, the typical generalized IDF relationship for a specific return period is as given in equation (2)

$$
\mathrm{i}=\frac{a}{\left(d^{v}+b\right)^{e}}
$$

where $a, b, e$ and $\mathrm{v}$ are non-negative coefficients. Thus, the equation that is more general: with $\mathrm{v}=1$ and $e=1$ will be Talbot equation; $\mathrm{v}=1$ and $b=0$ is Sherman; $e=1$ is Kimijima equation and $\mathrm{v}=1$ is Sherman. Equation (2) is an empirical formula that summarizes the experience from several studies.

There has been considerable attention and research on the IDF relationship: Hershfield [7] developed various rainfall contour maps to provide the design rain depths for various return periods and durations. Bell [8] proposed a generalized IDF formula using the one hour, 10 years rainfall depths; $\mathrm{P}_{1}{ }^{10}$, as an index. Chen [9] further developed a generalized IDF formula for any location in the United States using three base rainfall depths: $\mathrm{P}_{1}{ }^{10}, \mathrm{P}_{24}{ }^{10}, \mathrm{P}_{1}{ }^{100}$, which describe the geographical variation of rainfall. Kothyari and Garde [10] presented a relationship between rainfall intensity and $\mathrm{P}_{24}{ }^{2}$ for India.

The engineering application of rainfall intensity in the estimation of design discharge for flood control structures cannot be over-emphasized. The variability of the intensity, duration and frequency of rainstorm events necessitates location based development of intensity duration - frequency models. Therefore, this paper proposes the approach of development of different intensity - duration - frequency models using data from recording station. The research objective therefore is to develop and compare the exponential, logarithmic and power intensity duration frequency models for different return periods for Calabar.

\subsection{Study Area}

Calabar Metropolis lies between latitudes $04^{\circ} 45^{\prime}$ $30^{\prime \prime}$ North and $05^{\circ} 08^{\prime} 30^{\prime \prime}$ North of the Equator and longitudes $8^{\circ} 11^{\prime} 21^{\prime \prime}$ and $8^{\circ} 30^{\prime} 00^{\prime \prime}$ East of the Meridian. The town is flanked on its eastern and western borders by two large perennial streams viz: the Great Kwa River and the Calabar
River respectively. Calabar city lies in a peninsular between the two rivers, $56 \mathrm{~km}$ up the Calabar River away from the sea. Calabar has been described as an inter-fluvial settlement [11]. In some wet years $(1976,1978,1980,19$ 95, 1996, 1997, 1999, 2001, 2005, 2007 and 2008), rainfall reading have been observed to go up to over 3000mm (NIMET, [12]).

\section{Materials and Methods 2.1 Data Collection and Preparation}

The major material used in this study is rainfall data comprising of rainfall durations and intensities in Calabar. Rainfall data for twenty three years within the period 1983 to 2010 were obtained from Nigeria Meteorological Agency (NIMET) Calabar, Nigeria. This office is the data base for most climatic parameters in Calabar and its environ. The data preparation process involved sorting the data according to years, rainfall intensities and durations. The annual maximum monthly rainfall intensity values for each year of record were selected for frequency analysis.

\subsection{Data Analysis}

Various approaches can be used for the analysis of data to be employed in IDF plots. For this study, the graphical method basically was employed. Rainfall durations were abstracted from the rainfall event data obtained from the data source. The intensity was obtained by dividing the depth (amount) of rain by the duration as given by the expression;

$\mathrm{I}=\frac{R}{t}$

Where $\mathrm{I}$, is the rainfall intensity in $\mathrm{mm} / \mathrm{hr}, \mathrm{R}$, is the amount of rainfall in $\mathrm{mm}$, and $\mathrm{t}$ is the duration of the rainfall in hours. The monthly maximum intensities for each year were collated to form an annual series which were ranked in decreasing order of magnitude. The ranked annual maximum series of rainfall intensity values were analyzed aspresented in Table 1.

The return period or recurrence interval (T) is the average number of years during which a flood of given magnitude will be equaled or exceeded once is computed by one of the following methods [14]. Weibull's method was adopted in this study. Weibull's equation is expressed in equation (4) below.

$\mathrm{T}=\frac{\mathrm{n}+1}{\mathrm{~m}}$ 
where, $\mathrm{T}$ is the recurrence interval in years; $\mathrm{n}$ is the highest rank; and $\mathrm{m}$ is the rank value of each rainfall intensity.

\subsection{Model Development}

The exponential model employed to fit rainfall intensity values is in the form as presented in equation (5)

$i=a e^{b t}$

The logarithmic model employed to fit rainfall intensity values is in the form as presented in equation (6)

$i=a \ln (\mathrm{t})+\mathrm{b}$

The power model employed to fit rainfall intensity values is in the form as presented in equation (7) $i=a t^{b}$

where $\mathrm{i}$ is the rainfall intensity $(\mathrm{mm} / \mathrm{hr}), \mathrm{t}$ is the duration (minutes) a and $\mathrm{b}$ are regional constants and $\mathrm{e}=2.718$. The exponential, logarithmic and power intensity duration frequency models were developed by plotting the monthly rainfall intensity values against duration for each corresponding year using Microsoft Excel software. The data used for the models generation are as shown in Table 2 .

The models derived are presented in Table 3. The models obtained were therefore used in the forecast of rainfall intensities of different durations $(t=2,5,10,15,30,60,120,240$ and 320 minutes). The intensities obtained for the aforementioned durations are shown in Tables 4, 5 and 6 for the exponential, logarithmic and power models respectively.

Chow [15] has shown that most frequency functions can be generalized to eq. (8)

$X_{T}=\bar{X}+K_{T} s$

Where $K_{\mathrm{T}}$ is frequency factor for return period using Extreme Value Type 1 (Gumbel) distribution, $\mathrm{s}$ is the standard deviation of rainfall intensities and $\bar{X}$ is the mean rainfall intensities $X_{T}$ is the rainfall intensity for a given return period.

The frequency factor is calculated for each return period using Extreme Value Type 1 distribution as derived by Chow [9] and shown in eq. (9) below

$$
K_{T}=-\frac{\sqrt{6}}{\pi}\left\{0.5772+\ln \left[\ln \left(\frac{T}{T-1}\right)\right]\right\}
$$

where $\mathrm{T}$ is return period

Using eq. (8) and eq. (9), exponential, logarithmic and power intensity duration frequency models are developed for return periods between 2 years and 100 years using rainfall intensity data for durations of $2,5,10,15,30,60,120,240$ and 320 minutes as shown in figures 1,2 and 3.

Table 1: Annual maximum monthly rainfall in Calabar[13]

\begin{tabular}{|c|c|c|c|c|c|c|c|}
\hline \multirow[t]{2}{*}{ Year } & \multirow{2}{*}{$\begin{array}{l}\text { Depth } \\
(\mathrm{mm})\end{array}$} & \multicolumn{2}{|c|}{ Duration } & \multirow{2}{*}{$\begin{array}{l}\text { Intensity } \\
(\mathrm{mm} / \mathrm{hr})\end{array}$} & \multirow[t]{2}{*}{ Rank } & \multirow{2}{*}{$\begin{array}{c}\text { Return } \\
\text { Period } \\
\text { (T)(years) }\end{array}$} & \multirow{2}{*}{$\begin{array}{c}\text { Probability } \\
(1 / \mathrm{T})\end{array}$} \\
\hline & & hours & minutes & & & & \\
\hline 2009 & 9.33 & 0.03 & 1.8 & 311.0 & 1 & 24.00 & 0.04 \\
\hline 2001 & 5.04 & 0.02 & 1.2 & 252.0 & 2 & 12.50 & 0.08 \\
\hline 1986 & 16.92 & 0.09 & 5.4 & 188.0 & 3 & 8.67 & 0.12 \\
\hline 1985 & 10.92 & 0.06 & 3.6 & 182.0 & 4 & 6.75 & 0.15 \\
\hline 2002 & 39.96 & 0.27 & 16.2 & 148.0 & 5 & 5.60 & 0.18 \\
\hline 2006 & 10.29 & 0.07 & 4.2 & 147.0 & 6 & 4.83 & 0.21 \\
\hline 1989 & 18.08 & 0.16 & 9.6 & 113.0 & 7 & 4.29 & 0.23 \\
\hline 1990 & 11.22 & 0.11 & 6.6 & 102.0 & 8 & 3.88 & 0.26 \\
\hline 1994 & 14.76 & 0.15 & 9.0 & 98.4 & 9 & 3.56 & 0.28 \\
\hline 1996 & 54.38 & 0.57 & 34.2 & 95.4 & 10 & 3.30 & 0.30 \\
\hline 1983 & 11.28 & 0.14 & 8.4 & 80.6 & 11 & 3.09 & 0.32 \\
\hline 1988 & 45.06 & 0.59 & 35.4 & 76.4 & 12 & 2.92 & 0.34 \\
\hline 2005 & 68.71 & 0.91 & 54.6 & 75.5 & 13 & 2.77 & 0.36 \\
\hline 2003 & 13.25 & 0.18 & 10.8 & 73.6 & 14 & 2.64 & 0.38 \\
\hline 1995 & 94.8 & 1.37 & 82.2 & 69.2 & 15 & 2.53 & 0.39 \\
\hline 1992 & 75.68 & 1.1 & 66 & 68.8 & 16 & 2.44 & 0.41 \\
\hline 1997 & 106.83 & 1.76 & 105.6 & 60.7 & 17 & 2.35 & 0.43 \\
\hline 2004 & 19.79 & 0.34 & 20.4 & 58.2 & 18 & 2.28 & 0.44 \\
\hline 2000 & 64.98 & 1.24 & 74.4 & 52.4 & 19 & 2.21 & 0.45 \\
\hline 2010 & 19.72 & 0.4 & 24.0 & 49.3 & 20 & 2.15 & 0.47 \\
\hline 2007 & 15.91 & 0.36 & 21.6 & 44.2 & 21 & 2.10 & 0.48 \\
\hline 1991 & 100.1 & 2.56 & 153.6 & 39.1 & 22 & 2.05 & 0.49 \\
\hline 2008 & 64.96 & 1.77 & 106.2 & 36.7 & 23 & 2.00 & 0.50 \\
\hline
\end{tabular}


Table 2: Annual maximum daily rainfall in Calabar

\begin{tabular}{|c|c|c|c|c|c|c|c|c|c|c|c|c|c|}
\hline \multirow[b]{2}{*}{ Month } & \multirow[b]{2}{*}{ Unit } & \multicolumn{12}{|c|}{ Year } \\
\hline & & $\begin{array}{l}\text { No } \\
\stackrel{8}{\circ} \\
\infty\end{array}$ & $\begin{array}{l}\vec{b} \\
\stackrel{\infty}{\omega}\end{array}$ & $\begin{array}{l}\text { N } \\
\text { Oे } \\
\text { W }\end{array}$ & $\begin{array}{l}\text { 옹 } \\
\stackrel{\circ}{\circ}\end{array}$ & $\begin{array}{l}\text { 용 } \\
\text { \& }\end{array}$ & 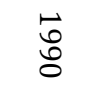 & $\begin{array}{l}\text { ㅇ } \\
\text { ᄋ }\end{array}$ & $\begin{array}{l}\text { 옹 } \\
\text { ᄋ̆ }\end{array}$ & $\begin{array}{l}\overrightarrow{0} \\
\infty \\
\sim \\
\sim\end{array}$ & $\begin{array}{l}\vec{b} \\
\infty \\
\infty\end{array}$ & $\begin{array}{l}\bullet \\
\underset{\infty}{\infty} \\
\infty\end{array}$ & $\begin{array}{l}\text { N } \\
\text { ᄋ } \\
\mathbb{1}\end{array}$ \\
\hline \multirow{2}{*}{ JAN. } & Intensity $(\mathrm{mm} / \mathrm{hr})$ & 29.8 & 0.0 & 73.6 & 0.0 & 20.0 & 13.9 & 0.0 & 18.8 & 28.1 & 0.0 & 26.6 & 1.0 \\
\hline & Depth (mm) & 25.0 & 0.0 & 13.0 & 0.0 & 120.0 & 48.0 & 0.0 & 240.0 & 19.0 & 0.0 & 54.0 & 51.0 \\
\hline \multirow{2}{*}{ FEB. } & Intensity $(\mathrm{mm} / \mathrm{hr})$ & 4.5 & 2.9 & 17.2 & 5.3 & 0.0 & 0.0 & 11.6 & 12.4 & 0.0 & 126.3 & 8.3 & 49.1 \\
\hline & Depth $(\mathrm{mm})$ & 133.0 & 21.0 & 154.0 & 130.0 & 0.0 & 0.0 & 85.0 & 25.0 & 0.0 & 10.0 & 38.0 & 20.0 \\
\hline \multirow{2}{*}{ MAR. } & Intensity $(\mathrm{mm} / \mathrm{hr})$ & 11.2 & 0.8 & 35.7 & 12.2 & 14.8 & 8.3 & 14.0 & 53.4 & 14.1 & 22.8 & 7.4 & 27.0 \\
\hline & Depth $(\mathrm{mm})$ & 20.0 & 144.0 & 53.0 & 250.0 & 30.0 & 87.0 & 420.0 & 30.0 & 16.0 & 30.0 & 529.0 & 3.0 \\
\hline \multirow{2}{*}{ APR. } & Intensity $(\mathrm{mm} / \mathrm{hr})$ & 31.2 & 8.4 & 33.3 & 21.1 & 7.9 & 57.5 & 18.9 & 25.4 & 106.0 & 16.6 & 76.8 & 25.7 \\
\hline & Depth (mm) & 26.0 & 40.0 & 85.0 & 95.0 & 110.0 & 12.0 & 45.0 & 79.0 & 80.0 & 56.0 & 45.0 & 102.0 \\
\hline \multirow{2}{*}{ MAY } & Intensity $(\mathrm{mm} / \mathrm{hr})$ & 24.3 & 80.6 & 36.3 & 252.0 & 43.9 & 14.1 & 44.2 & 91.0 & 182.0 & 14.4 & 39.1 & 13.3 \\
\hline & Depth (mm) & 57.0 & 11.0 & 58.0 & 4.0 & 20.0 & 73.0 & 16.0 & 10.0 & 11.0 & 118.0 & 20.0 & 63.0 \\
\hline \multirow{2}{*}{ JUNE } & Intensity $(\mathrm{mm} / \mathrm{hr})$ & 36.7 & 140.7 & 46.0 & 32.3 & 21.9 & 20.4 & 19.6 & 33.1 & 95.8 & 17.3 & 25.1 & 13.1 \\
\hline & Depth (mm) & 65.0 & 90.0 & 25.0 & 45.0 & 118.0 & 130.0 & 138.0 & 88.0 & 30.0 & 27.0 & 174.0 & 166.0 \\
\hline \multirow{2}{*}{ JULY } & Intensity $(\mathrm{mm} / \mathrm{hr})$ & 19.8 & 33.5 & 27.0 & 12.4 & 39.6 & 17.1 & 24.1 & 146.7 & 31.2 & 185.0 & 20.0 & 58.2 \\
\hline & Depth $(\mathrm{mm})$ & 204.0 & 10.0 & 128.0 & 120.0 & 70.0 & 270.0 & 122.0 & 10.0 & 49.0 & 17.0 & 15.0 & 20.0 \\
\hline \multirow{2}{*}{ AUG. } & Intensity $(\mathrm{mm} / \mathrm{hr})$ & 18.0 & 26.7 & 30.7 & 90.9 & 24.3 & 25.3 & 42.4 & 16.1 & 57.6 & 188.2 & 16.0 & 21.4 \\
\hline & Depth $(\mathrm{mm})$ & 118.0 & 20.0 & 9.0 & 45.0 & 220.0 & 185.0 & 22.0 & 60.0 & 23.0 & 47.0 & 18.0 & 50.0 \\
\hline \multirow{2}{*}{ SEPT. } & Intensity $(\mathrm{mm} / \mathrm{hr})$ & 8.6 & 14.5 & 34.3 & 13.2 & 18.5 & 101.7 & 18.3 & 12.2 & 7.0 & 6.4 & 15.0 & 40.5 \\
\hline & Depth (mm) & 282.0 & 35.0 & 112.0 & 45.0 & 110.0 & 11.0 & 220.0 & 59.0 & 477.0 & 15.0 & 233.0 & 20.0 \\
\hline \multirow{2}{*}{ OCT. } & Intensity $(\mathrm{mm} / \mathrm{hr})$ & 20.8 & 9.2 & 23.7 & 43.1 & 52.4 & 47.6 & 9.4 & 33.6 & 107.7 & 64.8 & 74.8 & 30.5 \\
\hline & Depth $(\mathrm{mm})$ & 105.0 & 8.0 & 85.0 & 33.0 & 65.0 & 50.0 & 177.0 & 40.0 & 18.0 & 15.0 & 36.0 & 70.0 \\
\hline \multirow{2}{*}{ NOV. } & Intensity $(\mathrm{mm} / \mathrm{hr})$ & 14.4 & 4.6 & 10.8 & 87.1 & 22.1 & 57.5 & 19.1 & 13.0 & 42.4 & 60.5 & 18.9 & 12.6 \\
\hline & Depth (mm) & 30.0 & 66.0 & 10.0 & 31.0 & 48.0 & 95.0 & 239.0 & 284.0 & 37.0 & 12.0 & 47.0 & 20.0 \\
\hline \multirow{2}{*}{ DEC. } & Intensity $(\mathrm{mm} / \mathrm{hr})$ & 13.8 & 62.8 & 0.0 & 4.0 & 22.9 & 1.2 & 19.6 & 3.0 & 6.6 & 0.0 & 15.7 & 23.1 \\
\hline & Depth (mm) & 183.0 & 28.0 & 0.0 & 25.0 & 120.0 & 30.0 & 32.0 & 20.0 & 30.0 & 0.0 & 84.0 & 70.0 \\
\hline \multirow[b]{2}{*}{ Month } & & & & & & & & Year & & & & & \\
\hline & Unit & & $\stackrel{\bullet}{\bullet}$ & $\begin{array}{l}\text { No } \\
\text { ○ } \\
\text { ํ. }\end{array}$ & $\begin{array}{l}\text { 응 } \\
\text { ○ }\end{array}$ & $\begin{array}{l}\vec{b} \\
\text { ஜे }\end{array}$ & $\begin{array}{l}\overrightarrow{0} \\
\text { 닌 }\end{array}$ & $\begin{array}{l}\vec{b} \\
\stackrel{0}{0}\end{array}$ & 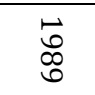 & 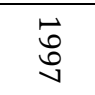 & $\begin{array}{l}\text { No } \\
\text { ○ } \\
\text { N }\end{array}$ & $\begin{array}{l}\text { 긍 } \\
\text { ○ }\end{array}$ & $\begin{array}{l}\vec{b} \\
\stackrel{0}{0}\end{array}$ \\
\hline & Intensity $(\mathrm{mm} / \mathrm{h}$ & & 0.0 & 11.5 & 17.1 & 1.6 & 0.0 & 5.0 & 0.0 & 18.8 & 0.0 & 3.3 & 15.5 \\
\hline JAN. & Depth (mm) & & 0.0 & 172.0 & 101.0 & 90.0 & 0.0 & 175.0 & 0.0 & 115.0 & 0.0 & 22.0 & 50.0 \\
\hline & Intensity $(\mathrm{mm} / \mathrm{h}$ & & 17.0 & 7.2 & 49.3 & 0.2 & 4.1 & 0.0 & 0.0 & 0.0 & 10.2 & 77.1 & 19.8 \\
\hline FEB. & Depth $(\mathrm{mm})$ & & 26.0 & 165.0 & 18.0 & 9.5 & 212.0 & 0.0 & 0.0 & 0.0 & 37.0 & 4.0 & 20.0 \\
\hline & Intensity $(\mathrm{mm} / \mathrm{h}$ & & 7.9 & 48.6 & 38.6 & 0.1 & 63.5 & 17.3 & 15.7 & 30.5 & 31.5 & 14.1 & 20.8 \\
\hline MAR. & Depth (mm) & & 20.0 & 126.0 & 13.0 & 210.0 & 10.0 & 580.0 & 86.0 & 37.0 & 49.0 & 102.0 & 19.0 \\
\hline & Intensity $(\mathrm{mm} / \mathrm{h}$ & & 19.9 & 20.7 & 27.1 & 20.4 & 26.0 & 3.3 & 22.3 & 41.5 & 29.9 & 12.9 & 26.7 \\
\hline APR. & Depth (mm) & & 65.0 & 88.0 & 27.0 & 220.0 & 81.0 & 202.0 & 204.0 & 125.0 & 78.0 & 130.0 & 40.0 \\
\hline MAY & Intensity $(\mathrm{mm} / \mathrm{h}$ & & 27.1 & 40.9 & 26.3 & 13.3 & 13.5 & 98.4 & 56.4 & 40.9 & 21.5 & 28.7 & 7.5 \\
\hline MAY & Depth (mm) & & 150.0 & 114.0 & 134.0 & 25.0 & 14.0 & 15.0 & 7.0 & 45.0 & 82.0 & 14.0 & 78.0 \\
\hline & Intensity $(\mathrm{mm} / \mathrm{h}$ & & 24.4 & 75.5 & 35.7 & 95.4 & 21.7 & 26.4 & 85.5 & 48.8 & 17.7 & 15.9 & 26.2 \\
\hline JUNE & Depth (mm) & & 290.0 & 69.0 & 132.0 & 54.0 & 55.0 & 15.0 & 13.0 & 15.0 & 50.0 & 99.0 & 84.0 \\
\hline UUY & Intensity $(\mathrm{mm} / \mathrm{h}$ & & 21.3 & 30.2 & 28.8 & 3.1 & 25.1 & 86.7 & 113.3 & 20.5 & 22.2 & 311.4 & 68.8 \\
\hline$\pi$ & Depth (mm) & & 75.0 & 90.0 & 65.0 & 175.0 & 90.0 & 9.0 & 18.0 & 82.0 & 30.0 & 10.0 & 75.0 \\
\hline & Intensity $(\mathrm{mm} / \mathrm{h}$ & & 39.1 & 39.1 & 40.0 & 0.5 & 24.1 & 35.2 & 20.5 & 60.7 & 19.4 & 30.0 & 11.7 \\
\hline AUG. & Depth (mm) & & 100.0 & 90.0 & 3.0 & 70.0 & 49.0 & 30.0 & 97.0 & 107.0 & 20.0 & 3.0 & 282.0 \\
\hline & Intensity $(\mathrm{mm} / \mathrm{h}$ & & 31.4 & 51.7 & 23.6 & 21.0 & 69.2 & 11.3 & 24.5 & 6.0 & 147.8 & 36.1 & 26.6 \\
\hline SEPT & Depth $(\mathrm{mm})$ & & 35.0 & 7.0 & 5.7 & 252.0 & 95.0 & 43.0 & 51.0 & 26.0 & 40.0 & 20.0 & 365.0 \\
\hline 0 & Intensity $(\mathrm{mm} / \mathrm{h}$ & & 10.7 & 25.8 & 30.9 & 38.8 & 25.5 & 30.0 & 16.9 & 2.9 & 52.0 & 50.4 & 45.2 \\
\hline U & Depth $(\mathrm{mm})$ & & 35.0 & 135.0 & 40.0 & 34.0 & 101.0 & 12.0 & 40.0 & 10.0 & 50.0 & 7.0 & 50.0 \\
\hline & Intensity $(\mathrm{mm} / \mathrm{h}$ & & 9.6 & 34.8 & 12.0 & 37.4 & 37.6 & 21.8 & 4.1 & 14.9 & 19.7 & 23.8 & 10.9 \\
\hline & Depth (mm) & & 10.0 & 87.0 & 36.0 & 62.0 & 15.0 & 102.0 & 133.0 & 50.0 & 45.0 & 166.0 & 163.0 \\
\hline & Intensity $(\mathrm{mm} / \mathrm{h}$ & & 8.6 & 19.9 & 8.6 & 0.5 & 20.1 & 0.0 & 0.0 & 24.9 & 8.8 & 0.0 & 0.5 \\
\hline DEC. & Depth $(\mathrm{mm})$ & & 123.0 & 84.0 & 250.0 & 79.0 & 205.0 & 0.0 & 0.0 & 14.0 & 15.0 & 0.0 & 13.0 \\
\hline
\end{tabular}


Table 3: Intensity-Duration Models for Calabar

\begin{tabular}{|c|c|c|c|}
\hline Year & Exponential Model & Logarithmic Model & Power Model \\
\hline 2009 & $\begin{array}{c}i=58.1291 e^{-0.003 t} \\
R^{2}=0.4931\end{array}$ & $\begin{array}{c}i=-25.06 \ln (t)+152.87 \\
R^{2}=0.4122\end{array}$ & $\begin{array}{c}i=147.54 t^{-0.424} \\
R^{2}=0.6835\end{array}$ \\
\hline 2001 & $\begin{array}{c}i=48.391 e^{-0.002 t} \\
R^{2}=0.4504\end{array}$ & $\begin{array}{c}i=-31.43 \ln (t)+201.52 \\
R^{2}=0.8484\end{array}$ & $\begin{array}{c}i=335.29 t^{-0.546} \\
R^{2}=0.8445\end{array}$ \\
\hline 1986 & $\begin{array}{c}i=67.15 e^{-0.005 t} \\
R^{2}=0.3915\end{array}$ & $\begin{array}{c}i=-35.03 \ln (t)+197.87 \\
R^{2}=0.6389\end{array}$ & $\begin{array}{c}i=427.17 t^{-0.65} \\
R^{2}=0.7911\end{array}$ \\
\hline 1985 & $\begin{array}{c}i=47.837 e^{-5 E-04 t} \\
R^{2}=0.2972\end{array}$ & $\begin{array}{c}i=-23.32 \ln (t)+154.69 \\
R^{2}=0.6023\end{array}$ & $\begin{array}{c}i=319.53 t^{-0.53} \\
R^{2}=0.7491\end{array}$ \\
\hline 2002 & $\begin{array}{c}i=56.523 e^{-0.007 t} \\
R^{2}=0.3667\end{array}$ & $\begin{array}{c}i=-43.7 \ln (t)+235.03 \\
R^{2}=0.7098\end{array}$ & $\begin{array}{c}i=913.45 t^{-0.787} \\
R^{2}=0.599\end{array}$ \\
\hline 2006 & $\begin{array}{c}i=34.998 e^{-0.001 t} \\
R^{2}=0.2227\end{array}$ & $\begin{array}{c}i=-21.31 \ln (t)+139.59 \\
R^{2}=0.8034\end{array}$ & $\begin{array}{c}i=239.38 t^{-0.484} \\
R^{2}=0.6739\end{array}$ \\
\hline 1989 & $\begin{aligned} i= & 43.955 e^{-0.001 t} \\
& R^{2}=0.66\end{aligned}$ & $\begin{array}{c}i=-16.1 \ln (t)+114.27 \\
R^{2}=0.7588\end{array}$ & $\begin{array}{c}i=228.6 t^{-0.466} \\
R^{2}=0.8654\end{array}$ \\
\hline 1990 & $\begin{array}{c}i=54.499 e^{-0.002 t} \\
R^{2}=0.7869\end{array}$ & $\begin{array}{c}i=-15.87 \ln (t)+115.16 \\
R^{2}=0.847\end{array}$ & $\begin{array}{c}i=387.06 t^{-0.568} \\
R^{2}=0.6632\end{array}$ \\
\hline 1994 & $\begin{array}{c}i=37.407 e^{-7 E-04 t} \\
R^{2}=0.6535\end{array}$ & $\begin{array}{c}i=-11.54 \ln (t)+90.331 \\
R^{2}=0.6717\end{array}$ & $\begin{array}{c}i=169.37 t^{-0.424} \\
R^{2}=0.822\end{array}$ \\
\hline 1996 & $\begin{array}{c}i=6.1723 e^{-2 E-05 t} \\
R^{2}=0.3397\end{array}$ & $\begin{array}{c}i=-8.146 \ln (t)+76.744 \\
R^{2}=0.5631\end{array}$ & $\begin{array}{c}i=1685.2 t^{-0.862} \\
R^{2}=0.8529\end{array}$ \\
\hline 1983 & $\begin{array}{c}i=21.591 e^{-3 E-04 t} \\
R^{2}=0.4417\end{array}$ & $\begin{array}{c}i=-12.6 \ln (t) 95.007 \\
R^{2}=0.3532\end{array}$ & $\begin{array}{c}i=380.73 t^{-0.681} \\
R^{2}=0.8189\end{array}$ \\
\hline 1988 & $\begin{array}{c}i=26.528 e^{-3 E-04 t} \\
R^{2}=0.2942\end{array}$ & $\begin{array}{c}i=-10.14 \ln (t)+79.993 \\
R^{2}=0.4362\end{array}$ & $\begin{array}{c}i=139.95 t^{-0.365} \\
R^{2}=0.5784\end{array}$ \\
\hline 2005 & $\begin{array}{c}i=46.464 e^{-0.001 t} \\
R^{2}=0.8098\end{array}$ & $\begin{array}{c}i=-11.57 \ln (t)+93.838 \\
R^{2}=0.6153\end{array}$ & $\begin{array}{c}i=254.35 t^{-0.422} \\
R^{2}=0.6811\end{array}$ \\
\hline 2003 & $\begin{array}{c}i=37.402 e^{-0.001 t} \\
R^{2}=0.1934\end{array}$ & $\begin{array}{c}i=-8.685 \ln (t)+72.513 \\
R^{2}=0.4092\end{array}$ & $\begin{array}{c}i=76.505 t^{-0.208} \\
R^{2}=0.253\end{array}$ \\
\hline 1995 & $\begin{array}{c}i=32.482 e^{-7 E-04 t} \\
R^{2}=0.6516\end{array}$ & $\begin{array}{c}i=-8.934 \ln (t)+74.155 \\
R^{2}=0.4818\end{array}$ & $\begin{array}{c}i=163.66 t^{-0.388} \\
R^{2}=0.6164\end{array}$ \\
\hline 1992 & $\begin{array}{c}i=36.643 e^{-0.002 t} \\
R^{2}=0.6127\end{array}$ & $\begin{array}{c}i=-8.829 \ln (t)+72.048 \\
R^{2}=0.4143\end{array}$ & $\begin{array}{c}i=531.45 t^{-0.644} \\
R^{2}=0.4647\end{array}$ \\
\hline 1997 & $\begin{array}{c}i=43.427 e^{-0.005 t} \\
R^{2}=0.2837\end{array}$ & $\begin{array}{c}i=-10.86 \ln (t)+79.891 \\
R^{2}=0.3148\end{array}$ & $\begin{array}{c}i=255 t^{-0.523} \\
R^{2}=0.2818\end{array}$ \\
\hline 2004 & $\begin{array}{c}i=30.991 e^{-0.001 t} \\
R^{2}=0.8576\end{array}$ & $\begin{array}{c}i=-7.368 \ln (t)+61.24 \\
R^{2}=0.5735\end{array}$ & $\begin{array}{c}i=205.5 t^{-0.499} \\
R^{2}=0.6321\end{array}$ \\
\hline 2000 & $\begin{array}{c}i=37.715 e^{-0.002 t} \\
R^{2}=0.5735\end{array}$ & $\begin{array}{c}i=-10.26 \ln (t)+80.584 \\
R^{2}=0.5839\end{array}$ & $\begin{array}{c}i=187.55 t^{-0.394} \\
R^{2}=0.5513\end{array}$ \\
\hline 2010 & $\begin{array}{c}i=31.228 e^{-8 E-04 t} \\
R^{2}=0.5511\end{array}$ & $\begin{array}{c}i=-5.021 \ln (t)+50.452 \\
R^{2}=0.4919\end{array}$ & $\begin{array}{c}i=67.907 t^{-0.222} \\
R^{2}=0.5142\end{array}$ \\
\hline 2007 & $\begin{array}{c}i=26.6 e^{-6 E-04 t} \\
R^{2}=0.4203\end{array}$ & $\begin{array}{c}i=-6.873 \ln (t)+60.439 \\
R^{2}=0.7544\end{array}$ & $\begin{array}{c}i=93.427 t^{-0.278} \\
R^{2}=0.7095\end{array}$ \\
\hline 1991 & $\begin{array}{c}i=18.799 e^{-3 E-04 t} \\
R^{2}=0.0215\end{array}$ & $\begin{array}{c}i=-0.964 \ln (t)+24.808 \\
R^{2}=0.0065\end{array}$ & $\begin{array}{c}i=21.31 t^{-0.04} \\
R^{2}=0.0038\end{array}$ \\
\hline 2008 & $\begin{array}{c}y=24.76 e^{-7 E-04 t} \\
R^{2}=0.6376\end{array}$ & $\begin{array}{c}i=-5.636 \ln (t)+50.802 \\
R^{2}=0.548\end{array}$ & $\begin{array}{c}i=120.38 t^{-0.353} \\
R^{2}=0.5651\end{array}$ \\
\hline
\end{tabular}


Table 4: Rainfall intensity values $(\mathrm{mm} / \mathrm{hr}$ ) of exponential models of various durations for Calabar

\begin{tabular}{cccccccccc}
\hline Years & $\begin{array}{c}2 \\
(\mathrm{mins})\end{array}$ & 5 (mins) & $10(\mathrm{mins})$ & $\begin{array}{c}15 \\
(\mathrm{mins})\end{array}$ & $\begin{array}{c}30 \\
\text { (mins) }\end{array}$ & $\begin{array}{c}60 \\
\text { (mins) }\end{array}$ & $\begin{array}{c}120 \\
(\mathrm{mins})\end{array}$ & $\begin{array}{c}240 \\
\text { (mins) }\end{array}$ & $\begin{array}{c}320 \\
(\mathrm{mins})\end{array}$ \\
\hline 2009 & 57.78 & 57.26 & 56.41 & 55.57 & 53.13 & 48.55 & 40.56 & 28.29 & 22.26 \\
\hline 2001 & 48.20 & 47.91 & 47.43 & 46.96 & 45.57 & 42.92 & 38.07 & 29.94 & 25.52 \\
\hline 1986 & 66.49 & 65.50 & 63.88 & 62.30 & 57.80 & 49.75 & 36.85 & 20.23 & 13.56 \\
\hline 1985 & 47.79 & 47.72 & 47.60 & 47.48 & 47.12 & 46.42 & 45.05 & 42.43 & 40.76 \\
\hline 2002 & 55.74 & 54.58 & 52.70 & 50.89 & 45.82 & 37.14 & 24.40 & 10.53 & 6.02 \\
\hline 2006 & 34.93 & 34.82 & 34.65 & 34.48 & 33.96 & 32.96 & 31.04 & 27.53 & 25.41 \\
\hline 1989 & 43.87 & 43.74 & 43.52 & 43.30 & 42.66 & 41.40 & 38.98 & 34.58 & 31.92 \\
\hline 1990 & 54.28 & 53.96 & 53.42 & 52.89 & 51.33 & 48.34 & 42.87 & 33.72 & 28.74 \\
\hline 1994 & 37.35 & 37.28 & 37.15 & 37.02 & 36.63 & 35.87 & 34.39 & 31.62 & 29.90 \\
\hline 1996 & 6.17 & 6.17 & 6.17 & 6.17 & 6.17 & 6.16 & 6.16 & 6.14 & 6.13 \\
\hline 1983 & 21.58 & 21.56 & 21.53 & 21.49 & 21.40 & 21.21 & 20.83 & 20.09 & 19.61 \\
\hline 1988 & 26.51 & 26.49 & 26.45 & 26.41 & 26.29 & 26.05 & 25.59 & 24.69 & 24.10 \\
\hline 2005 & 46.37 & 46.23 & 46.00 & 45.77 & 45.09 & 43.76 & 41.21 & 36.55 & 33.74 \\
\hline 2003 & 37.33 & 37.22 & 37.03 & 36.85 & 36.30 & 35.22 & 33.17 & 29.42 & 27.16 \\
\hline 1995 & 32.44 & 32.37 & 32.26 & 32.14 & 31.81 & 31.15 & 29.86 & 27.46 & 25.96 \\
\hline 1992 & 36.50 & 36.28 & 35.92 & 35.56 & 34.51 & 32.50 & 28.82 & 22.67 & 19.32 \\
\hline 1997 & 42.99 & 42.35 & 41.31 & 40.29 & 37.38 & 32.17 & 23.83 & 13.08 & 8.77 \\
\hline 2004 & 30.93 & 30.84 & 30.68 & 30.53 & 30.08 & 29.19 & 27.49 & 24.38 & 22.50 \\
\hline 2000 & 37.56 & 37.34 & 36.97 & 36.60 & 35.52 & 33.45 & 29.67 & 23.34 & 19.89 \\
\hline 2010 & 31.18 & 31.10 & 30.98 & 30.86 & 30.49 & 29.76 & 28.37 & 25.77 & 24.17 \\
\hline 2007 & 26.57 & 26.52 & 26.44 & 26.36 & 26.13 & 25.66 & 24.75 & 23.03 & 21.95 \\
\hline 1991 & 18.79 & 18.77 & 18.74 & 18.71 & 18.63 & 18.46 & 18.13 & 17.49 & 17.08 \\
\hline 2008 & 24.73 & 24.68 & 24.59 & 24.51 & 24.25 & 23.75 & 22.77 & 20.94 & 19.80 \\
\hline & & & & & & & & &
\end{tabular}

Table 5: Rainfall intensity values $(\mathrm{mm} / \mathrm{hr}$ ) of logarithmic models of various durations for Calabar

\begin{tabular}{cccccccccc}
\hline Years & $\begin{array}{c}2 \\
(\mathrm{mins})\end{array}$ & 5 (mins) & $\begin{array}{c}10 \\
(\mathrm{mins})\end{array}$ & $\begin{array}{c}15 \\
(\mathrm{mins})\end{array}$ & $\begin{array}{c}30 \\
\text { (mins) }\end{array}$ & $\begin{array}{c}60 \\
\text { (mins) }\end{array}$ & $\begin{array}{c}120 \\
\text { (mins) }\end{array}$ & $\begin{array}{c}240 \\
\text { (mins) }\end{array}$ & $\begin{array}{c}320 \\
\text { (mins) }\end{array}$ \\
\hline 2009 & 135.50 & 112.54 & 95.17 & 85.01 & 67.64 & 50.27 & 32.90 & 15.53 & 8.32 \\
\hline 2001 & 179.71 & 150.92 & 129.13 & 116.39 & 94.60 & 72.81 & 51.03 & 29.24 & 20.20 \\
\hline 1986 & 173.59 & 141.49 & 117.21 & 103.01 & 78.73 & 54.45 & 30.16 & 5.88 & -4.19 \\
\hline 1985 & 218.87 & 197.50 & 181.33 & 171.88 & 155.71 & 139.55 & 123.39 & 107.22 & 100.51 \\
\hline 2002 & 204.74 & 164.70 & 134.41 & 116.69 & 86.40 & 56.11 & 25.82 & -4.47 & -17.05 \\
\hline 1906 & 124.82 & 105.29 & 90.52 & 81.88 & 67.11 & 52.34 & 37.57 & 22.80 & 16.67 \\
\hline 1990 & 103.11 & 88.36 & 77.20 & 70.67 & 59.51 & 48.35 & 37.19 & 26.03 & 21.40 \\
\hline 1994 & 82.33 & 71.76 & 63.76 & 59.08 & 51.08 & 43.08 & 35.08 & 27.08 & 23.76 \\
\hline 1996 & 71.10 & 63.63 & 57.99 & 54.68 & 49.04 & 43.39 & 37.75 & 32.10 & 29.76 \\
\hline 1983 & 86.27 & 74.73 & 65.99 & 60.89 & 52.15 & 43.42 & 34.68 & 25.95 & 22.33 \\
\hline 2005 & 85.82 & 75.22 & 67.20 & 62.51 & 54.49 & 46.47 & 38.45 & 30.43 & 27.10 \\
\hline 2003 & 66.49 & 58.54 & 52.52 & 48.99 & 42.97 & 36.95 & 30.93 & 24.91 & 22.42 \\
\hline 1995 & 67.96 & 59.78 & 53.58 & 49.96 & 43.77 & 37.58 & 31.38 & 25.19 & 22.62 \\
\hline 1992 & 65.93 & 57.84 & 51.72 & 48.14 & 42.02 & 35.90 & 29.78 & 23.66 & 21.12 \\
\hline
\end{tabular}




\begin{tabular}{cccccccccc}
\hline Years & $\begin{array}{c}2 \\
\text { (mins) }\end{array}$ & 5 (mins) & $\begin{array}{c}10 \\
\text { (mins) }\end{array}$ & $\begin{array}{c}15 \\
\text { (mins) }\end{array}$ & $\begin{array}{c}30 \\
\text { (mins) }\end{array}$ & $\begin{array}{c}60 \\
\text { (mins) }\end{array}$ & $\begin{array}{c}120 \\
\text { (mins) }\end{array}$ & $\begin{array}{c}240 \\
\text { (mins) }\end{array}$ & $\begin{array}{c}320 \\
\text { (mins) }\end{array}$ \\
\hline 1997 & 72.36 & 62.41 & 54.88 & 50.48 & 42.95 & 35.43 & 27.90 & 20.37 & 17.25 \\
\hline 2004 & 56.13 & 49.38 & 44.27 & 41.29 & 36.18 & 31.07 & 25.97 & 20.86 & 18.74 \\
\hline 2000 & 73.47 & 64.07 & 56.96 & 52.80 & 45.69 & 38.58 & 31.46 & 24.35 & 21.40 \\
\hline 2010 & 46.97 & 42.37 & 38.89 & 36.86 & 33.38 & 29.90 & 26.42 & 22.94 & 21.50 \\
\hline 2007 & 55.68 & 49.38 & 44.61 & 41.83 & 37.06 & 32.30 & 27.53 & 22.77 & 20.79 \\
\hline 1991 & 24.14 & 23.26 & 22.59 & 22.20 & 21.53 & 20.86 & 20.19 & 19.52 & 19.25 \\
\hline 2008 & 46.90 & 41.73 & 37.82 & 35.54 & 31.63 & 27.73 & 23.82 & 19.91 & 18.29 \\
\hline
\end{tabular}

Table 6: Rainfall intensity values ( $\mathrm{mm} / \mathrm{hr}$ ) of power models of various durations for Calabar

\begin{tabular}{cccccccccc}
\hline Years & $\begin{array}{c}2 \\
\text { (mins) }\end{array}$ & $\begin{array}{c}5 \\
\text { (mins) }\end{array}$ & $\begin{array}{c}10 \\
\text { (mins) }\end{array}$ & $\begin{array}{c}15 \\
\text { (mins) }\end{array}$ & $\begin{array}{c}30 \\
\text { (mins) }\end{array}$ & $\begin{array}{c}60 \\
\text { (mins) }\end{array}$ & $\begin{array}{c}120 \\
\text { (mins) }\end{array}$ & $\begin{array}{c}240 \\
\text { (mins) }\end{array}$ & $\begin{array}{c}320 \\
\text { (mins) }\end{array}$ \\
\hline 2009 & 109.97 & 74.57 & 55.58 & 46.80 & 34.88 & 26.00 & 19.38 & 14.44 & 12.79 \\
\hline 2001 & 229.65 & 139.25 & 95.37 & 76.43 & 52.35 & 35.86 & 24.56 & 16.82 & 14.38 \\
\hline 1986 & 272.23 & 150.06 & 95.63 & 73.48 & 46.82 & 29.84 & 19.02 & 12.12 & 10.05 \\
\hline 1985 & 221.29 & 136.16 & 94.30 & 76.06 & 52.68 & 36.48 & 25.27 & 17.50 & 15.02 \\
\hline 2002 & 529.39 & 257.39 & 149.17 & 108.42 & 62.83 & 36.41 & 21.10 & 12.23 & 9.75 \\
\hline 2006 & 171.15 & 109.85 & 78.54 & 64.54 & 46.15 & 33.00 & 23.59 & 16.87 & 14.68 \\
\hline 1989 & 165.50 & 107.98 & 78.18 & 64.72 & 46.85 & 33.92 & 24.56 & 17.78 & 15.55 \\
\hline 1990 & 261.09 & 155.15 & 104.66 & 83.13 & 56.08 & 37.83 & 25.52 & 17.21 & 14.62 \\
\hline 1994 & 126.24 & 85.60 & 63.80 & 53.72 & 40.04 & 29.85 & 22.25 & 16.58 & 14.68 \\
\hline 1996 & 927.18 & 420.86 & 231.55 & 163.25 & 89.82 & 49.42 & 27.19 & 14.96 & 11.67 \\
\hline 1983 & 237.47 & 127.24 & 79.36 & 60.21 & 37.56 & 23.43 & 14.61 & 9.11 & 7.49 \\
\hline 1988 & 108.67 & 77.78 & 60.39 & 52.08 & 40.44 & 31.40 & 24.38 & 18.93 & 17.04 \\
\hline 2005 & 189.84 & 128.96 & 96.26 & 81.12 & 60.55 & 45.19 & 33.73 & 25.18 & 22.30 \\
\hline 2003 & 66.23 & 54.74 & 47.39 & 43.56 & 37.71 & 32.65 & 28.26 & 24.47 & 23.05 \\
\hline 1995 & 125.07 & 87.65 & 66.98 & 57.23 & 43.73 & 33.42 & 25.54 & 19.52 & 17.46 \\
\hline 1992 & 340.09 & 188.51 & 120.63 & 92.91 & 59.46 & 38.05 & 24.35 & 15.58 & 12.95 \\
\hline 1997 & 177.46 & 109.90 & 76.48 & 61.86 & 43.05 & 29.96 & 20.85 & 14.51 & 12.48 \\
\hline 2004 & 145.41 & 92.05 & 65.13 & 53.20 & 37.65 & 26.64 & 18.85 & 13.34 & 11.55 \\
\hline 2000 & 142.73 & 99.48 & 75.70 & 64.53 & 49.11 & 37.37 & 28.44 & 21.64 & 19.32 \\
\hline 2010 & 58.22 & 47.51 & 40.73 & 37.22 & 31.91 & 27.36 & 23.46 & 20.11 & 18.87 \\
\hline 2007 & 77.05 & 59.73 & 49.26 & 44.01 & 36.29 & 29.93 & 24.69 & 20.36 & 18.79 \\
\hline 1991 & 20.73 & 19.98 & 19.43 & 19.12 & 18.60 & 18.09 & 17.60 & 17.11 & 16.92 \\
\hline 2008 & 94.25 & 68.21 & 53.40 & 46.28 & 36.24 & 28.37 & 22.21 & 17.39 & 15.71 \\
\hline & & & & & & & & &
\end{tabular}




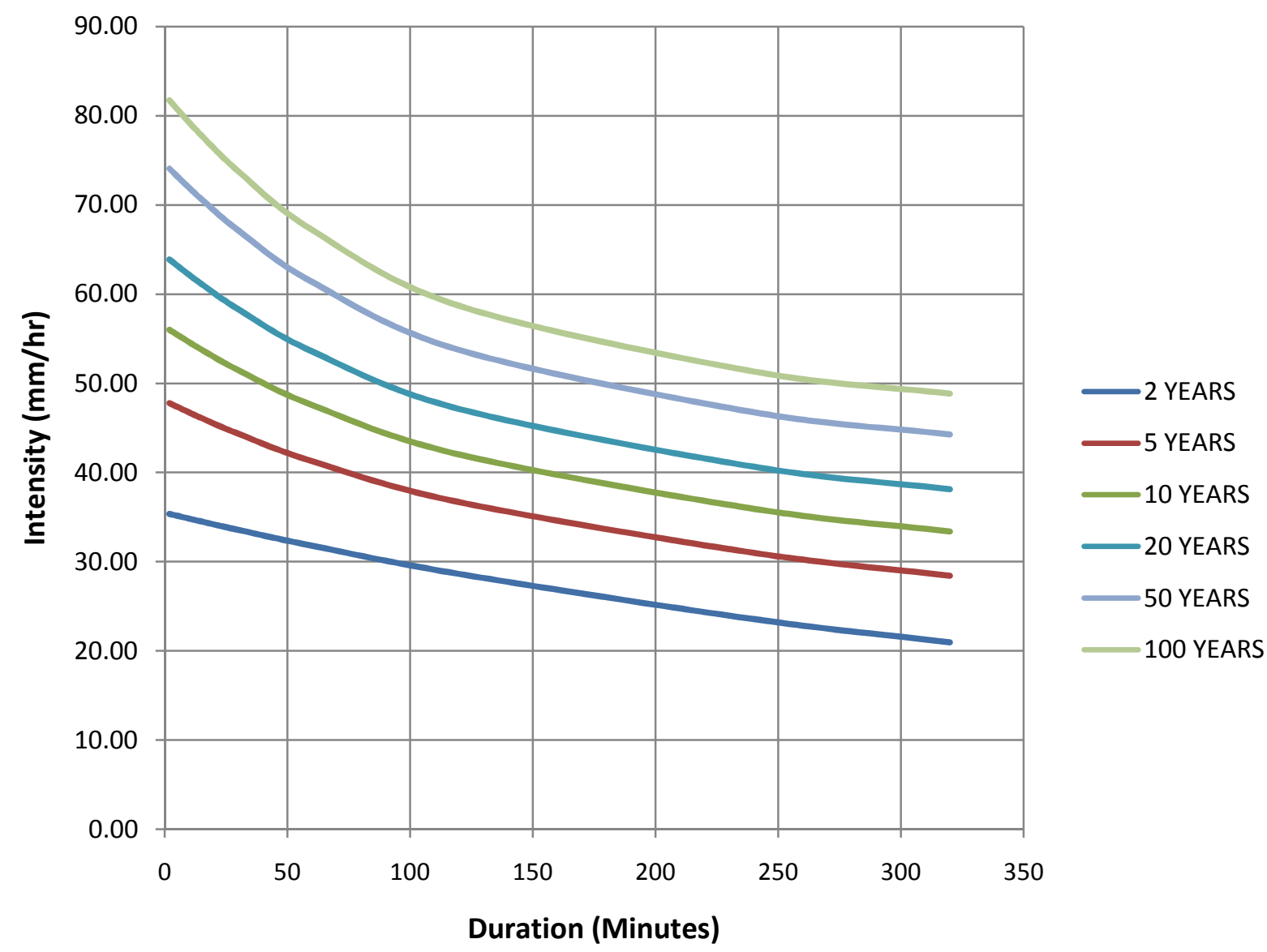

Figure 1: Intensity Duration Frequency Curve of Exponential Models for Calabar

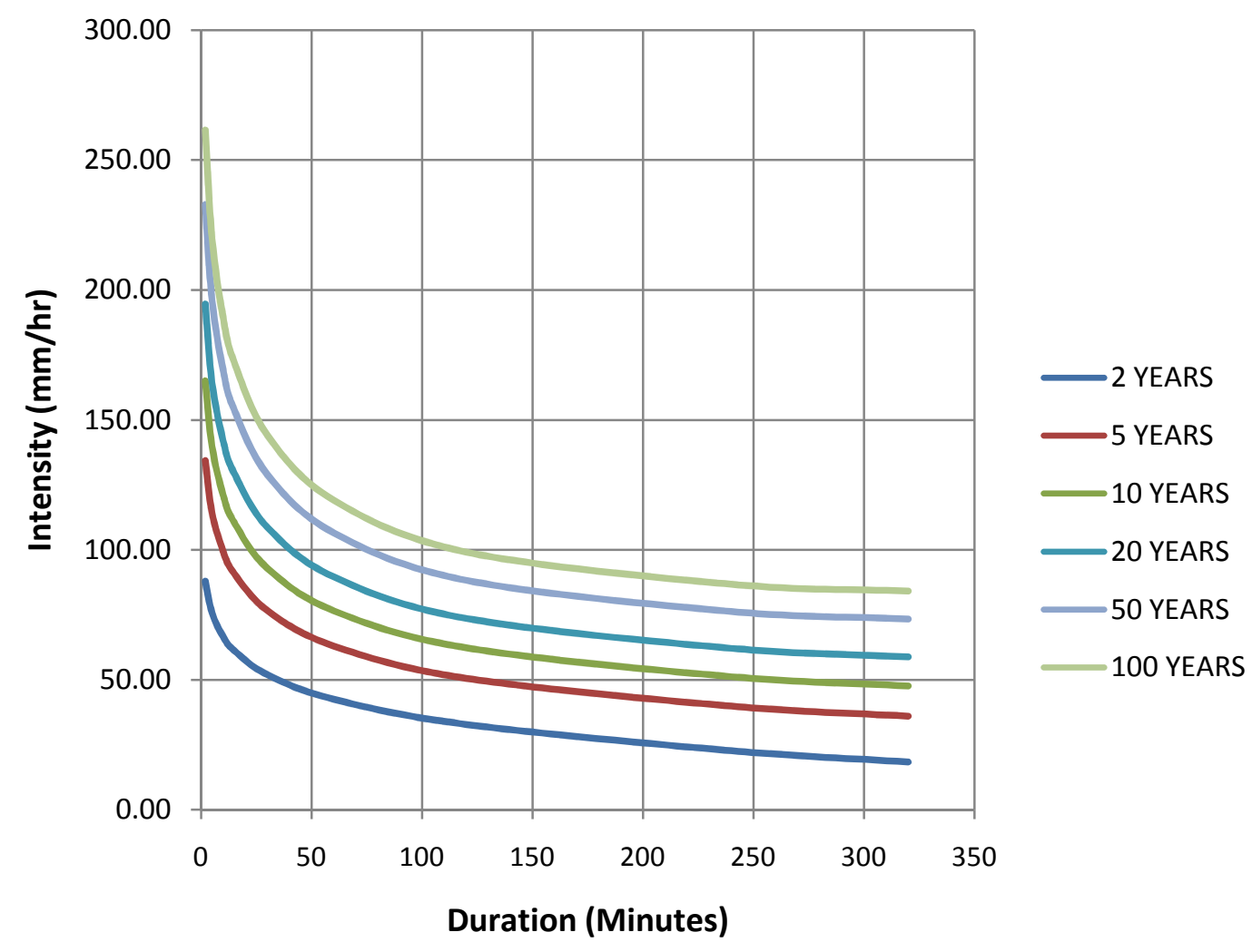

Figure 2: Intensity Duration Frequency Curves of Logarithmic Models for Calabar 


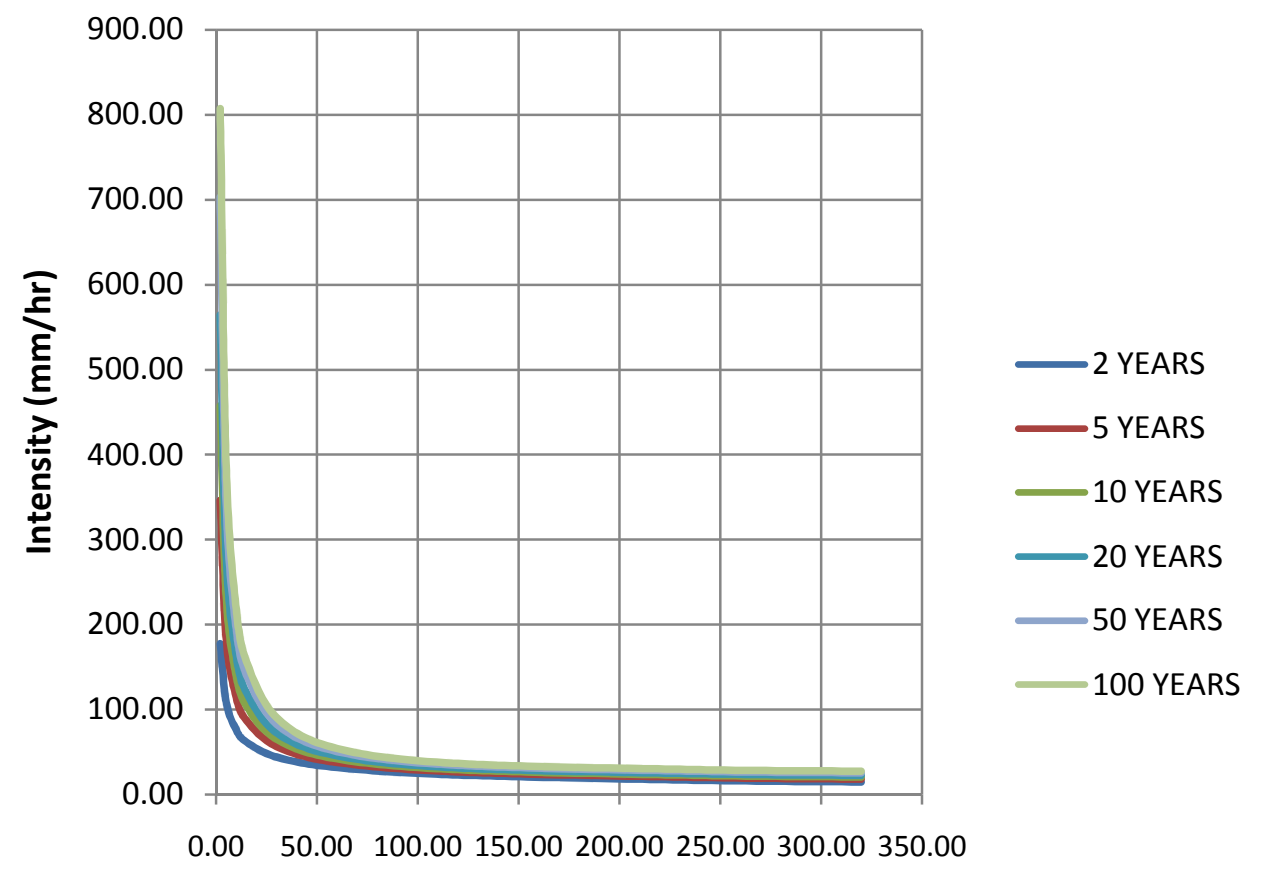

Duration (Minutes)

Figure 3: Intensity Duration Frequency Curves of Power Models for Calabar

\section{Results and discussion}

The results for the comparison of the exponential, logarithmic and power intensity duration frequency models for different return periods are presented in the plots of Figures 1, 2 and 3 as shown.

For durations between 2 to 10 minutes, the power model gave results that have relatively higher average intensity values than the logarithmic and exponential models. On the other hand, the logarithmic model gave results that have relatively higher average intensity values than the exponential and power models for durations between 30 to 320 minutes. The logarithmic models gave results with higher intensity values for the 15 minutes duration than the exponential and power models; except for the 2 years return period for which the power model was slightly higher.

A physical inspection of the intensity - duration frequency plot of the three models (Figures 1, 2 and 3) reveal that the curves of exponential and logarithmic models have better gradation than the power model.

\section{Conclusion}

This study has been conducted to develop and compare the exponential, logarithmic and power intensity duration frequency models for different return periods. In general, comparing the predicted results by the three models to the measured intensity values (Table 1), the logarithmic model yielded the closest intensity values to the measured intensities. The use of the results of the power model for the prediction of intensities for small duration rainstorm would lead to oversizing the design of such hydraulic structures implying undesired high cost burden. The use of the results of the exponential model for the prediction of intensities would lead to under sizing the design of such hydraulic structures implying failure in design of such structures. Therefore the logarithmic model yields moderate intensity-duration-frequency models that would ensure adequate sizing and forecast of relevant hydraulic structures in Calabar.

\section{References}

1. Egbuniwe, N., Variations in the statistical measures of mean rainfall. Nigerian Journal of Technology, Vol. 2, No.1, 1978, pp. 19 - 22.

2. Eze, H.I., Effect of rainfall intensity and energy on gully development in northeastern Enugu State, Nigeria. Nigerian Journal of Technology, Vol. 26, No.1, 2007, pp. $91-96$.

3. Bernard M.M., "Formulas for rainfall intensities of long duration", Transactions, ASCE, 96(Paper No.1801), 1932, pp.592-624. 
4. El-Sayed, E. A., Generation of Rainfall Intensity Duration Frequency Curves For Ungauged Sites. Nile Basin Water Science \& Engineering Journal, 4(1), 2011, pp.112-124.

5. Okonkwo G.I. and Mbajiorgu, C.C., Rainfall Intensity-Duration-Frequency Analyses for South Eastern Nigeria", Agricultural Engineering International: the CIGR Ejournal. Manuscript 1304. Vol. XII, 2010.

6. Chow V.T., D.R. Maidment and L.W.Mays, "Applied Hydrology", McGraw- Hill, 1988.

7. Hershfield, D.M., "Estimating the Probable Maximum precipitation" Journal of the Hydraulic Division, Proceeding of the ASCE, HY5, 1961, pp. 99-116.

8. Bell, F.C., Generalized rainfall duration frequency relationships. Journal of Hydraulic Div.,ASCE, 95(1), 1969, pp.311-327.

9. Chen, C.L., Rainfall intensity-duration -frequency formulas, Journal of Hydraulic Engineering, ASCE, 109(12), 1983, pp. 1603-1621.

10. Kothyari, U.C. and Garde, R.J., Rainfall intensity duration frequency formula for India, J. Hydr. Engrg., ASCE, 118(2), 1992, pp.323-336.
11. Ugbong, I.A., An Analysis of Runoff flow, Channel Characteristics \& Flood \& Erosion Menace in the Calabar Drainage Basin. An MsC Research, Dept. of Geography and Regional Planning, University of Calabar, 2000.

12. Antigha, R.E.E., Urban Storm Water Drainage Systems Modeling for Calabar Metropolis, Cross River State, Nigeria: PhD Dissertation (in press), Dept. of Agric \&Environmental Engineering, Rivers State University of Science \& Technology, Port Harcourt, Nigeria, 2012.

13. Antigha, R.E. and Ogarekpe, N.M., "Development of Intensity Duration Frequency Curves for Calabar Metropolis, South- South, Nigeria", The International Journal of Engineering and Science (THE IJES), 2(3), 2013, pp. 39 - 42.

14. Raghunath, H.M., "Hydrology", Wiley Eastern Limited, 1986.

15. Chow, V. T. A general formula for hydrologic frequency analysis, Trans Am. Geophysical Union, Vol. 32, No. 2, 1951, pp. 231-237.

16. Chow, V. T. Frequency analysis of hydrologic data with special application to rainfall intensities, Bulletin. No. 414, University of Illinois Eng. Expt Station, 1953 